\title{
RULED SURFACES IN ELLIPTIC SPACE
}

\section{CURTIS M. FULTON}

Bonnet's theorem on ruled surfaces [2, p. 449] deals solely with intrinsic properties if an intrinsic definition of the line of striction is adopted. Contrasting this, our aim is to define the parameter of distribution and the line of striction in relation to the enveloping space and to show that they have the usual properties. Attention will be called occasionally to the changes required to treat the hyperbolic case. The two theorems hold in hyperbolic space with minor variations and follow by formally analogous proofs. In fact, in the first one $K \neq-1$ must be assumed and in the second the hyperbolic tangent of the distance has to be used. Notation and terminology will largely be taken from [1].

Let " $D$ be the standard connection on the Euclidean 4-space such that " $D_{V} W=\left(V W^{i}\right) e_{i}$ where $e_{i}, i=1,2,3,4$ constitutes the natural frame field. Our elliptic 3-space is represented by the unit hypersphere $\langle x, x\rangle=1$, so the position vector $x$ serves as unit normal and we have " $D_{V} x=V$. If now ' $D$ stands for the induced connection on the elliptic space we find for vectors tangent to it

$$
\text { " } D_{V} W={ }^{\prime} D_{V} W-\langle V, W\rangle x \text {. }
$$

In order to adjust this setup to hyperbolic space the metric $\langle$, would acquire one minus sign for the Minkowski 4-space, the imbedded hypersurface would be given by $\langle x, x\rangle=-1$, and the minus sign in (1) would change to plus. With the usual notation for the curvature tensor $[1$, p. 59] we get with the aid of (1).

$$
\text { ' } R(U, V) W=\langle V, W\rangle U-\langle U, W\rangle V .
$$

Denoting by $D$ the connection on a surface immersed in the elliptic 3 -space and by $N$ its unit normal vector field and defining the Weingarten map by $L(V)={ }^{\prime} D_{V} N$, we see in view of (2) that for vectors pertaining to the surface $[1$, p. 77] ' $R(U, V) W=R(U, V) W$ $-\langle L V, W\rangle L(U)+\langle L U, W\rangle L(V)$. This, in combination with (2), yields an expression that will allow us to compute the Gaussian curvature, namely

$$
\begin{aligned}
\langle U, R(U, V) V\rangle= & \langle U, U\rangle\langle V, V\rangle-\langle U, V\rangle^{2}+\langle L U, U\rangle\langle L V, V\rangle \\
& -\langle L U, V\rangle\langle L V, U\rangle .
\end{aligned}
$$

Received by the editors March 24, 1969. 
A geodesic of the elliptic space, defined by ' $D_{V} V=0$, on account of (1) satisfies " $D_{V} V=-x$ and is therefore represented by

$$
x=(\cos v) A+(\sin v) B,\langle A, A\rangle=\langle B, B\rangle=1,\langle A, B\rangle=0 .
$$

In the hyperbolic case there would appear hyperbolic functions in (4). We now wish to determine the common perpendicular to two lines $x=(\cos v) A+(\sin v) B$ and $x_{1}=\left(\cos v_{1}\right) A_{1}+\left(\sin v_{1}\right) B_{1}$ whose unit tangents are $V=(-\sin v) A+(\cos v) B$ and $V_{1}=\left(-\sin v_{1}\right) A_{1}+\left(\cos v_{1}\right) B_{1}$. As we easily infer from (4), the distance $E$ between two points $x, x_{1}$ may be found by means of $\cos E=\left\langle x, x_{1}\right\rangle$ and $x_{1}-(\cos E) x$ is a vector at $x$ along the line joining $x$ and $x_{1}$. We want this vector to be perpendicular to $V$, which condition reduces to $\left\langle V, x_{1}\right\rangle=0$. Similarly, $\left\langle V_{1}, x\right\rangle$ $=0$. In order to take care of these two perpendicularity conditions we use the abbreviations $a=\left\langle A, A_{1}\right\rangle-\left\langle B, B_{1}\right\rangle, b=\left\langle A, B_{1}\right\rangle+\left\langle B, A_{1}\right\rangle, c$ $=\left\langle A, A_{1}\right\rangle+\left\langle B, B_{1}\right\rangle, d=\left\langle A, B_{1}\right\rangle-\left\langle B, A_{1}\right\rangle$. This enables us to write two equivalent equations,

(5) $a \sin \left(v_{1}+v\right)-b \cos \left(v_{1}+v\right)=0, c \sin \left(v_{1}-v\right)-d \cos \left(v_{1}-v\right)=0$.

Setting $P^{2}=a^{2}+b^{2}, Q^{2}=c^{2}+d^{2}$, equations (5) imply

$$
\begin{aligned}
& a=P \cos \left(v_{1}+v\right), \quad b=P \sin \left(v_{1}+v\right), \quad c=Q \cos \left(v_{1}-v\right), \\
& d=Q \sin \left(v_{1}-v\right),
\end{aligned}
$$

where in general, the selection of signs for $P$ and $Q$ produces two solutions. Hyperbolic cosines appearing in (6) would allow for only one solution. Making use of (6) we obtain for the distance $E$ between the points $x$ and $x_{1}$ along the common perpendicular(s)

$$
\begin{aligned}
2 \cos E= & a \cos \left(v_{1}+v\right)+b \sin \left(v_{1}+v\right)+c \cos \left(v_{1}-v\right) \\
& +d \sin \left(v_{1}-v\right)=P+Q .
\end{aligned}
$$

In general, the two given lines are not coplanar and it will be useful to find the angle $\phi$ between the planes determined by the common perpendicular and either line. A plane, being a totally geodesic surface, allows parallel shifting of its normals in the enveloping space $[3$, p. 139]. Thus $\phi$ can be evaluated at any point of the common perpendicular and may be computed as the angle between $V$ and $V_{1}$. Hence,

$$
\begin{aligned}
2 \cos \phi= & -a \cos \left(v_{1}+v\right)-b \sin \left(v_{1}+v\right)+c \cos \left(v_{1}-v\right) \\
& +d \sin \left(v_{1}-v\right)=-P+Q .
\end{aligned}
$$

The parametrization of a ruled surface we wish to use takes the form $x=(\cos v) A(u)+(\sin v) B(u)$. As the generator corresponding to 
$u_{1}$ approaches the generator $u, c$ tends toward 2 and $d$ toward 0 , so that relations (6) tell us that $v_{1}$ approaches $v$. If we let $v^{*}$ designate the parameter of the central point into which the common perpendicular collapses we conclude from (6) that

$$
\tan 2 v^{*}=\lim b / a=2\left\langle A^{\prime}, B^{\prime}\right\rangle /\left(\left\langle A^{\prime}, A^{\prime}\right\rangle-\left\langle B^{\prime}, B^{\prime}\right\rangle\right) .
$$

There is no loss of generality if the line of striction, which is the locus of the central point, is taken as $v^{*}=0$; because of (9) $\left\langle A^{\prime}, B^{\prime}\right\rangle=0$ for this arrangement. In the elliptic case there are of course two central points on every generator. Geometrically, the parameter of distribution $p^{*}$ is defined as the limit as $u_{1}$ approaches $u$ of $E / \phi$. By means of standard methods using (7) and (8) one verifies for the square of this limit under the assumption that $v^{*}=0$.

$$
\begin{aligned}
\left(p^{*}\right)^{2} & =\lim E^{2} / \phi^{2}=\lim (P+Q-2) /(-P+Q-2) \\
& =\left[\left\langle A^{\prime}, A^{\prime}\right\rangle-\left\langle A, B^{\prime}\right\rangle^{2}\right] /\left[\left\langle B^{\prime}, B^{\prime}\right\rangle-\left\langle A, B^{\prime}\right\rangle^{2}\right] .
\end{aligned}
$$

Interestingly, Clifford parallels arise when the expressions in (7) and (8) are equal or differ in sign.

Returning to the parametrization of our ruled surface, we derive its natural frame field consisting of the vectors $U=(\cos v) A^{\prime}+(\sin v) B^{\prime}$ and $V=(-\sin v) A+(\cos v) B$. Moreover we will need the functions $g_{11}=\langle U, \quad U\rangle=\left\langle A^{\prime}, \quad A^{\prime}\right\rangle \cos ^{2} v+2\left\langle A^{\prime}, B^{\prime}\right\rangle \cos v \sin v+\left\langle B^{\prime}, B^{\prime}\right\rangle \sin ^{2} v$, $g_{12}=\langle U, V\rangle=\left\langle A^{\prime}, B\right\rangle, g_{22}=\langle V, V\rangle=1$. A vector $M$ normal to the surface is seen to be given by $\left\langle M, e_{i}\right\rangle=\left(e_{i}, x, U, V\right)=\left(e_{i}, A, U, B\right)$ where the latter symbols abbreviate fourth-order determinants. Squaring the determinant expression for $\langle M, M\rangle$ yields $\langle M, M\rangle=g_{11} g_{22}-\left(g_{12}\right)^{2}$ $=h^{2}$, where $h$ is meant to be positive. Thus the unit normal $N$ is expressed by $\left\langle N, e_{i}\right\rangle=h^{-1}\left(e_{i}, A, U, B\right)$. Since ' $D_{V} V=0,\langle L V, V\rangle$ vanishes in equation (3). On the other hand, by reason of (1) ' $D_{V} U$ $=(-\sin v) A^{\prime}+(\cos v) B^{\prime}+g_{12} x$. Also, $\langle L V, \quad U\rangle=-\left\langle D_{V}^{\prime} U, \quad N\right\rangle$ $=h^{-1}\left(A^{\prime}, A, B^{\prime}, B\right)$. As evidenced by (3) the curvature $K$ may now be written

$$
-h^{4}(K-1)=\left[\left\langle A^{\prime}, A^{\prime}\right\rangle-\left\langle A, B^{\prime}\right\rangle^{2}\right]\left[\left\langle B^{\prime}, B^{\prime}\right\rangle-\left\langle A, B^{\prime}\right\rangle^{2}\right]
$$

and (10) demonstrates that $p^{*}=0$ implies $K=1$, that is $K$ equals the curvature of the elliptic space [2, p. 456]. We observe from (11) that $V K=0$ is equivalent to $V h=0$ which in turn bears the same relation to $V g_{11}=0$. This gives the value of $v^{*}$ appearing in (9) and an intrinsic definition of the line of striction [2, p. 447] is now embodied in the

THEOREM. If $K \neq 1$ along a ruling, $V K=0$ characterizes the central point. 
Finally, let $N_{0}$ be the normal at the central point, taken as $v^{*}=0$, of a given ruling. Clearly, $h_{0}\left\langle N_{0}, e_{i}\right\rangle=\left(e_{i}, A, A^{\prime}, B\right)$, where $\left(h_{0}\right)^{2}$ $=\left\langle A^{\prime}, A^{\prime}\right\rangle-\left\langle A^{\prime}, B\right\rangle^{2}$. In order to evaluate the angle $\theta$ between $N$ and $N_{0}$ we proceed noting that $h h_{0}\left\langle N, N_{0}\right\rangle^{2}=\left(N_{0}, A, U, B\right)\left(N, A, A^{\prime}, B\right)$ which comes down to $h \cos \theta=h_{0} \cos v$. In addition we form $h^{2} \sin ^{2} \theta$ $=\left[\left\langle B^{\prime}, B^{\prime}\right\rangle-\left\langle A^{\prime}, B\right\rangle^{2}\right] \sin ^{2} v$ and conclude in view of $(10)$ that $\left(p^{*}\right)^{2}$ $\tan ^{2} \theta=\tan ^{2} v$ which proves the

THEOREM. The tangent of the angle through which the normal rotates as a point moves along a generator is proportional to the tangent of the distance from the central point to the moving point. The factor of proportionality is the reciprocal of the parameter of distribution [2, p. 458].

\section{REFERENCES}

1. N. J. Hicks, Notes on differential geometry, Van Nostrand, Princeton, N. J., 1965. MR 31 \#3936.

2. V. Hlavatý, Differentialgeometrie der Kurven und Flachen und Tensorrechnung, Noordhoff, Groningen, 1939. MR 1, 27.

3. C. E. Weatherburn, An introduction to Riemannian geometry, Cambridge Univ. Press, New York, 1957.

University of California, Davis 